J. Nonlinear Var. Anal. 5 (2021), No. 6, pp. 965-979

Available online at http://jnva.biemdas.com

https://doi.org/10.23952/jnva.5.2021.6.09

\title{
A HIERARCHICAL VARIATIONAL INEQUALITY PROBLEM FOR GENERALIZED DEMIMETRIC MAPPINGS WITH APPLICATIONS
}

\author{
MOHAMMAD ESLAMIAN \\ Department of Mathematics, University of Science and Technology of Mazandaran, Behshahr, Iran
}

\begin{abstract}
In this paper, we study a hierarchical variational inequality problem for two finite families of generalized demimetric mappings. We present a new and efficient iterative method for solving this problem and establish its strong convergence. As applications, we use our algorithm to solve the generalized multiple-set split feasibility problem, the triple hierarchical variational inequality problem, and the triple-hierarchical constrained optimization problem.

Keywords. Hierarchical variational inequality; Inverse-strongly monotone mappings; Generalized demimetric mappings; Split feasibility problem.
\end{abstract}

\section{INTRODUCTION}

Let $C$ be a nonempty closed convex subset of a real Hilbert space $\mathscr{H}$, and let $F: \mathscr{H} \rightarrow \mathscr{H}$ be an operator. The classical variational inequality problem (VIP) is formulated as follows:

$$
\text { Find } \quad x^{\star} \in C \quad \text { such that }\left\langle F x^{\star}, y-x^{\star}\right\rangle \geq 0, \quad \forall y \in C \text {. }
$$

The set of solutions of this problem is denoted by $V I(C, F)$. The theory of variational inequalities has emerged as an important tool to study a wide class of problems from science, engineering, social sciences, and management. It is a powerful unified methodology to study partial differential equations, optimization problems, optimal control problems, mathematical programming problems, financial management problems, equilibrium problems and so on; see, e.g., $[1,2,3,4,5]$ and the references therein.

Let $T: \mathscr{H} \rightarrow \mathscr{H}$ be a nonlinear mapping with nonempty fixed point set $F i x(T)$. The hierarchical variational inequality problem (in short, HVIP) is to find $x^{*} \in F i x(T)$ such that

$$
\left\langle F\left(x^{*}\right), y-x^{*}\right\rangle \geq 0, \quad \forall y \in F i x(T) .
$$

Many important application problems, such as signal recovery, power-control, bandwidth allocation, optimal control, and beamforming are special cases of hierarchical variational inequality problem; see, e.g., $[6,7,8,9]$ and the references therein.

Recently, Yamada [6] considered the following hybrid steepest-decent iterative method for solving the hierarchical variational inequality:

$$
x_{n+1}=\left(I-\mu \alpha_{n} F\right) T x_{n},
$$

${ }^{*}$ Corresponding author.

E-mail address: eslamian@mazust.ac.ir.

Received April 14, 2021; Accepted May 10, 2021.

(C)2021 Journal of Nonlinear and Variational Analysis 
where $F$ is a Lipschitzian continuous and strongly monotone mapping and $T$ is a nonexpansive mapping. Yamada [6] also considered the hierarchical variational inequality problem defined over the set of common fixed points of a finite family of nonexpansive mappings. The hybrid steepest-descent method has received great attention recent from many authors; see e.g., [10, 11] and the references therein.

In 2018, Kawasaki and Takahashi [12] introduced a new general class of mappings, called generalized demimetric mappings as follows.

Definition 1.1. Let $\zeta$ be a real number with $\zeta \neq 0$. A mapping $T: \mathscr{H} \rightarrow \mathscr{H}$ with $F i x(T) \neq \emptyset$ is said to be generalized demimetric if $\zeta\left\langle x-x^{\star}, x-T x\right\rangle \geq\|x-T x\|^{2}$, for all $x \in \mathscr{H}$ and $x^{\star} \in$ Fix $(T)$. This mapping $T$ is called the $\zeta$-generalized demimetric.

This class of mappings is fundamental because it includes many types of nonlinear mappings arising in applied mathematics and optimization (see [12,13] for details).

Now, let us mention inertial-type algorithms. Based on the heavy ball methods of the twoorder time dynamical system, Polyak [14] firstly proposed an inertial extrapolation as an acceleration process to solve the smooth convex minimization problem. The inertial algorithm is a two-step iterative method and the next iterate is defined by making use of the previous two iterates. Recently, a lot of researchers constructed fast iterative algorithms by using the inertial extrapolation; see, e.g., [15, 16, 17, 18, 19] and the references therein.

The purpose of this paper is to introduce an iterative algorithm, which combines the inertial acceleration method and the hybrid steepest-descent method for solving the hierarchical variational inequality problem with two finite families of generalized demimetric mappings. We obtain the strong convergence of the sequences generated by our iterative algorithm under certain weak conditions in Hilbert spaces. As applications, we use our algorithm to solve the generalized multiple-set split feasibility problem, the triple hierarchical variational inequality problem, and the triple-hierarchical constrained optimization problem.

\section{PRELIMinaries}

We use the following notations in the sequel:

$-\rightarrow$ for weak convergence and $\rightarrow$ for strong convergence.

Given a nonempty closed convex set $C \subset \mathscr{H}$, the mapping that assigns every point $x \in \mathscr{H}$, to its unique nearest point in $C$ is called the metric projection onto $C$ and is denoted by $P_{C}$, i.e., $P_{C}(x) \in C$ and $\left\|x-P_{C}(x)\right\|=\inf _{y \in C}\|x-y\|$. The metric projection $P_{C}$ is characterized by the fact that $P_{C}(x) \in C$ and $\left\langle y-P_{C}(x), x-P_{C}(x)\right\rangle \leq 0, \forall x \in \mathscr{H}, y \in C$. We refer to [20] for more properties of the metric projection.

Definition 2.1. A mapping $F: \mathscr{H} \rightarrow \mathscr{H}$ is said to be

- Lipschitz continuous with constant $L>0$ if $\|F(x)-F(y)\| \leq L\|x-y\|, \forall x, y \in \mathscr{H}$, further, if $L=1$, then $F$ is said to be nonexpansive;

- monotone if $\langle F(x)-F(y), x-y\rangle \geq 0, \forall x, y \in \mathscr{H}$;

- strongly monotone with constant $\beta>0$ if

$$
\langle F(x)-F(y), x-y\rangle \geq \beta\|x-y\|^{2}, \quad \forall x, y \in \mathscr{H} ;
$$

- inverse-strongly monotone with constant $\beta>0$, ( $\beta$-ism) if

$$
\langle F(x)-F(y), x-y\rangle \geq \beta\|F(x)-F(y)\|^{2}, \quad \forall x, y \in \mathscr{H} ;
$$


- strictly pseudo-contractive if there exists a constant $\beta \in[0,1)$ such that

$$
\|F x-F y\|^{2} \leq\|x-y\|^{2}+\beta\|(x-F x)-(y-F y)\|^{2}, \quad \forall x, y \in \mathscr{H} .
$$

Definition 2.2. Assume that $T: \mathscr{H} \rightarrow \mathscr{H}$ is a nonlinear mapping with $\operatorname{Fix}(T) \neq \emptyset$. Then $I-T$ is said to be demiclosed at zero if, for any $\left\{x_{n}\right\}$ in $\mathscr{H}$, the following implication holds:

$$
x_{n} \rightarrow x \text { and }(I-T) x_{n} \rightarrow 0 \Rightarrow x \in F i x(T) .
$$

Lemma 2.1. Let $\mathscr{H}$ be a real Hilbert space. Then, for all $x, y \in \mathscr{H},\|x+y\|^{2} \leq\|x\|^{2}+2\langle y, x+$ $y\rangle$.

Lemma 2.2. [12] Let $\mathscr{H}$ be a real Hilbert space and let $\theta$ be a real number with $\theta \neq 0$. Let $T: \mathscr{H} \rightarrow \mathscr{H}$ be a $\theta$-generalized demimetric mapping. Then the fixed point set Fix $(T)$ of $T$ is closed and convex.

Lemma 2.3. [20] Let $C$ be a nonempty closed and convex subset of real Hilbert space $\mathscr{H}$. Let $F: C \rightarrow \mathscr{H}$ be a strongly monotone and Lipschitz continuous mapping. Then $\operatorname{VIP}(C, F)$ consists only one point.

Lemma 2.4. [6] Let the operator $F: \mathscr{H} \rightarrow \mathscr{H}$ be l-Lipschitz continuous and $\delta$-strongly monotone with constants $l>0$ and $\delta>0$. Assume that $\gamma \in\left(0, \frac{2 \delta}{l^{2}}\right)$. For $\alpha \in(0,1)$, define $T_{\alpha}=I-$ $\alpha \gamma F$. Then, for all $x, y \in \mathscr{H},\left\|T_{\alpha} x-T_{\alpha} y\right\| \leq(1-\alpha \eta)\|x-y\|$, where $\eta=1-\sqrt{1-\gamma\left(2 \delta-\gamma l^{2}\right)}$ $\in(0,1)$.

Lemma 2.5. [21] Assume that $\left\{s_{n}\right\}$ is a sequence of nonnegative real numbers such that

$$
\left\{\begin{array}{l}
s_{n+1} \leq\left(1-\eta_{n}\right) s_{n}+\eta_{n} \delta_{n}, \quad n \geq 0, \\
s_{n+1} \leq s_{n}-\rho_{n}+\zeta_{n}, \quad n \geq 0,
\end{array}\right.
$$

where $\left\{\eta_{n}\right\}$ is a sequence in $(0,1),\left\{\rho_{n}\right\}$ is a sequence of nonnegative real numbers, and $\left\{\delta_{n}\right\}$ and $\left\{\zeta_{n}\right\}$ are two sequences in $\mathbb{R}$ such that

(i) $\sum_{n=1}^{\infty} \eta_{n}=\infty$,

(ii) $\lim _{n \rightarrow \infty} \zeta_{n}=0$

(iii) $\lim _{k \rightarrow \infty} \rho_{n_{k}}=0$, implies limsup $\operatorname{sum}_{k \rightarrow \infty} \delta_{n_{k}} \leq 0$ for any subsequence $\left\{n_{k}\right\} \subset\{n\}$.

Then $\lim _{n \rightarrow \infty} s_{n}=0$.

Lemma 2.6. [22] Let $F$ be a $\beta$-inverse-strongly monotone mapping with domain dom $(F)$ for some $\beta>0$. Let $C$ be a closed and convex subset of $\operatorname{dom}(F)$. Suppose that $\Gamma=F^{-1}(0) \cap C \neq \emptyset$, where $F^{-1}(0)$ denotes the set of zero points of $F$. Then $\operatorname{VI}(C, F)=\Gamma$.

\section{The Algorithm And Its Convergence AnAlysis}

In this section, we present our algorithm for solving the hierarchical variational inequality problem.

Theorem 3.1. Let $\mathscr{H}$ be a Hilbert space. Let, for each $i \in\{1,2, \ldots, m\}, \zeta^{(i)} \neq 0$ and $T^{(i)}: \mathscr{H} \rightarrow$ $\mathscr{H}$ be a $\zeta^{(i)}$-generalized demimetric mapping such that $I-T^{(i)}$ is demiclosed at 0 . Let, for each $j \in\{1,2, \ldots, k\}, \vartheta^{(j)} \neq 0$ and $S^{(j)}: \mathscr{H} \rightarrow \mathscr{H}$ be a $\vartheta^{(j)}$-generalized demimetric mapping such that $I-S^{(j)}$ is demiclosed at 0 . Suppose that $\Omega=\bigcap_{i=1}^{m} F i x\left(T^{(i)}\right) \cap \bigcap_{j=1}^{k} F i x\left(S^{(j)}\right) \neq \emptyset$. Let the 
operator $F: \mathscr{H} \rightarrow \mathscr{H}$ be l-Lipschitz continuous and $\delta$-strongly monotone with constants $l>0$ and $\delta>0$. Assume that $\gamma \in\left(0, \frac{2 \delta}{l^{2}}\right)$ and $\alpha^{*}>0$. Let $\left\{x_{n}\right\}$ be a sequence defined by

$$
\left\{\begin{array}{l}
x_{1}, x_{0} \in \mathscr{H} \text { is chosen arbitrarily, } \\
w_{n}=x_{n}+\alpha_{n}^{*}\left(x_{n}-x_{n-1}\right) \\
y_{n}=w_{n}+\sum_{i=1}^{m} a^{(i)} l^{(i)} \theta_{n}^{(i)}\left(T^{(i)} w_{n}-w_{n}\right) \\
z_{n}=y_{n}+\sum_{j=1}^{k} b^{(j)} r^{(j)} \delta_{n}^{(j)}\left(S^{(j)} y_{n}-y_{n}\right), \\
x_{n+1}=\left(I-\gamma \beta_{n} F\right) z_{n}, \quad n \geq 1
\end{array}\right.
$$

where $l^{(i)}=\frac{\zeta^{(i)}}{\left|\zeta^{(i)}\right|}, r^{(j)}=\frac{\vartheta^{(j)}}{\left|\vartheta^{(j)}\right|}$, and $0 \leq \alpha_{n}^{*} \leq \bar{\alpha}_{n}$ such that

$$
\bar{\alpha}_{n}=\left\{\begin{array}{l}
\min \left\{\frac{\varepsilon_{n}}{\left\|x_{n}-x_{n-1}\right\|}, \alpha^{*}\right\}, \quad x_{n} \neq x_{n-1} \\
\alpha^{*}, \quad \text { otherwise. }
\end{array}\right.
$$

Let the sequences $\left\{\beta_{n}\right\},\left\{\varepsilon_{n}\right\},\left\{a^{(i)}\right\},\left\{b^{(j)}\right\},\left\{\theta_{n}^{(i)}\right\}$, and $\left\{\delta_{n}^{(j)}\right\}$ satisfy the following conditions:

(i) $\left\{\beta_{n}\right\} \subset(0,1), \lim _{n \rightarrow \infty} \beta_{n}=0$, and $\sum_{n=0}^{\infty} \beta_{n}=\infty$;

(ii) $a^{(i)}, b^{(j)} \in(0,1]$ and $\sum_{i=1}^{m} a^{(i)}=\sum_{j=1}^{k} b^{(j)}=1$;

(iii) $0<c^{(i)} \leq \theta_{n}^{(i)}<2 \frac{l^{(i)}}{\zeta^{(i)}}$, for $i \in\{1,2, \ldots, m\}$;

(iv) $0<d^{(j)} \leq \delta_{n}^{(j)}<2 \frac{r^{(j)}}{\vartheta^{(j)}}$ for $j \in\{1,2, \ldots, k\}$;

(v) $\varepsilon_{n}>0$ and $\lim _{n \rightarrow \infty} \frac{\varepsilon_{n}}{\beta_{n}}=0$.

Then, $\left\{x_{n}\right\}$ converges strongly to the unique solution $x^{\star} \in V I(\Omega, F)$.

Proof. From Lemma 2.2, we know that $\operatorname{Fix}\left(T^{(i)}\right)$ and $\operatorname{Fix}\left(S^{(j)}\right)$ are closed and convex. Therefore $\Omega=\bigcap_{i=1}^{m} F i x\left(T^{(i)}\right) \cap \bigcap_{j=1}^{k} F i x\left(S^{(j)}\right)$ is closed and convex. Since $\Omega$ is nonempty, closed and convex, we have from Lemma 2.3 that $\operatorname{VI}(\Omega, F)$ has a unique solution. We denote by $x^{\star} \in \mathscr{H}$ the unique solution of $\operatorname{VI}(\Omega, F)$. Since $\|\cdot\|^{2}$ is convex and, for each $i=1,2, . ., m, T^{(i)}: \mathscr{H} \rightarrow \mathscr{H}$ is a $\zeta^{(i)}$-generalized demimetric mapping, we obtain that

$$
\begin{aligned}
\left\|y_{n}-x^{\star}\right\|^{2} \leq & \sum_{i=1}^{m} a^{(i)}\left\|w_{n}+l^{(i)} \theta_{n}^{(i)}\left(T^{(i)} w_{n}-w_{n}\right)-x^{\star}\right\|^{2} \\
= & \sum_{i=1}^{m} a^{(i)}\left[\left\|w_{n}-x^{\star}\right\|^{2}+2\left\langle w_{n}-x^{\star}, l^{(i)} \theta_{n}^{(i)}\left(T^{(i)} w_{n}-w_{n}\right)\right\rangle\right. \\
& \left.+\left\|l^{(i)} \theta_{n}^{(i)}\left(T^{(i)} w_{n}-w_{n}\right)\right\|^{2}\right] \\
\leq & \sum_{i=1}^{m} a^{(i)}\left[\left\|w_{n}-x^{\star}\right\|^{2}-2\left(l^{(i)} \theta_{n}^{(i)}\right)\left(\frac{1}{\zeta(i)}\right)\left\|T^{(i)} w_{n}-w_{n}\right\|^{2}\right. \\
& \left.+\left(\theta_{n}^{(i)}\right)^{2}\left\|T^{(i)} w_{n}-w_{n}\right\|^{2}\right] \\
= & \left\|w_{n}-x^{\star}\right\|^{2}-\sum_{i=1}^{m} a^{(i)} \theta_{n}^{(i)}\left(\frac{2 l^{(i)}}{\zeta(i)}-\theta_{n}^{(i)}\right)\left\|T^{(i)} w_{n}-w_{n}\right\|^{2} .
\end{aligned}
$$


For each $j=1,2, . ., k$, since $S^{(j)}: \mathscr{H} \rightarrow \mathscr{H}$ is a $\vartheta^{(j)}$ - generalized demimetric mapping, we conclude that

$$
\begin{aligned}
\left\|z_{n}-x^{\star}\right\|^{2} & =\left\|y_{n}+\sum_{j=1}^{k} b^{(j)} r^{(j)} \delta_{n}^{(j)}\left(S^{(j)} y_{n}-y_{n}\right)-x^{\star}\right\|^{2} \\
& \leq\left\|y_{n}-x^{\star}\right\|^{2}-\sum_{j=1}^{k} b^{(j)} \delta_{n}^{(j)}\left(\frac{2 r^{(j)}}{\vartheta^{(j)}}-\delta_{n}^{(j)}\right)\left\|S^{(j)} y_{n}-y_{n}\right\|^{2} .
\end{aligned}
$$

From inequalities (3.2) and (3.3), we have

$$
\begin{aligned}
\left\|z_{n}-x^{\star}\right\|^{2} \leq & \left\|w_{n}-x^{\star}\right\|^{2}-\sum_{i=1}^{m} a^{(i)} \theta_{n}^{(i)}\left(\frac{2 l^{(i)}}{\zeta(i)}-\theta_{n}^{(i)}\right)\left\|T^{(i)} w_{n}-w_{n}\right\|^{2} \\
& -\sum_{j=1}^{k} b^{(j)} \delta_{n}^{(j)}\left(\frac{2 r^{(j)}}{\vartheta(j)}-\delta_{n}^{(j)}\right)\left\|S^{(j)} y_{n}-y_{n}\right\|^{2} .
\end{aligned}
$$

From condition (iii), condition ( $i v)$, and inequality (3.4), we arrive at

$$
\left\|z_{n}-x^{\star}\right\| \leq\left\|w_{n}-x^{\star}\right\| \text {. }
$$

We have $\alpha_{n}^{*}\left\|x_{n}-x_{n-1}\right\| \leq \varepsilon_{n}$ for all $n$, which together with $\lim _{n \rightarrow \infty} \frac{\varepsilon_{n}}{\beta_{n}}=0$, implies that

$$
\lim _{n \rightarrow \infty} \frac{\alpha_{n}^{*}}{\beta_{n}}\left\|x_{n}-x_{n-1}\right\|=0 .
$$

It follows that there exists a constant $M_{1}>0$ such that

$$
\frac{\alpha_{n}^{*}}{\beta_{n}}\left\|x_{n}-x_{n-1}\right\| \leq M_{1} \text {. }
$$

From the definition of $w_{n}$, we obtain

$$
\begin{aligned}
\left\|w_{n}-x^{\star}\right\| & \leq\left\|x_{n}-x^{\star}\right\|+\alpha_{n}^{*}\left\|x_{n}-x_{n-1}\right\| \\
& \leq\left\|x_{n}-x^{\star}\right\|+\beta_{n} M_{1} .
\end{aligned}
$$

From inequality (3.5) and inequality (3.6), we have

$$
\left\|z_{n}-x^{\star}\right\| \leq\left\|w_{n}-x^{\star}\right\| \leq\left\|x_{n}-x^{\star}\right\|+\beta_{n} M_{1} .
$$

Utilizing Lemma 2.4 and above inequality, we conclude that

$$
\begin{aligned}
\left\|x_{n+1}-x^{\star}\right\| & =\left\|\left(I-\beta_{n} \gamma F\right) z_{n}-\left(I-\beta_{n} \gamma F\right) x^{\star}-\beta_{n} \gamma F x^{\star}\right\| \\
& \leq\left\|\left(I-\beta_{n} \gamma F\right) z_{n}-\left(I-\beta_{n} \gamma F\right) x^{\star}\right\|+\beta_{n} \gamma\left\|F x^{\star}\right\| \\
& \leq\left(1-\beta_{n} \eta\right)\left\|z_{n}-x^{\star}\right\|+\beta_{n} \gamma\left\|F x^{\star}\right\| \\
& \leq\left(1-\beta_{n} \eta\right)\left\|x_{n}-x^{\star}\right\|+\beta_{n} M_{1}+\beta_{n} \gamma\left\|F x^{\star}\right\| \\
& \leq\left(1-\beta_{n} \eta\right)\left\|x_{n}-x^{\star}\right\|+\beta_{n} \eta\left[\frac{M_{1}+\gamma\left\|F x^{\star}\right\|}{\eta}\right] \\
& \leq \max \left\{\left\|x_{n}-x^{\star}\right\|, \frac{M_{1}+\gamma\left\|F x^{\star}\right\|}{\eta}\right\}, \\
& \leq \cdots \leq \max \left\{\left\|x_{1}-x^{\star}\right\|, \frac{M_{1}+\gamma\left\|F x^{\star}\right\|}{\eta}\right\},
\end{aligned}
$$


where $\eta=1-\sqrt{1-\gamma\left(2 \delta-\gamma l^{2}\right)} \in(0,1)$. This implies that $\left\{x_{n}\right\}$ is bounded. We also obtain $\left\{y_{n}\right\}$ and $\left\{z_{n}\right\}$ are bounded. It follows that

$$
\begin{aligned}
\left\|w_{n}-x^{\star}\right\|^{2} & \left.=\| x_{n}+\alpha_{n}^{*}\left(x_{n}-x_{n-1}\right)-x^{\star}\right) \|^{2} \\
& \leq\left\|x_{n}-x^{\star}\right\|^{2}+\left(\alpha_{n}^{*}\right)^{2}\left\|x_{n}-x_{n-1}\right\|^{2}+2 \alpha_{n}^{*}\left\langle x_{n}-x^{\star}, x_{n}-x_{n-1}\right\rangle \\
& \leq\left\|x_{n}-x^{\star}\right\|^{2}+\left(\alpha_{n}^{*}\right)^{2}\left\|x_{n}-x_{n-1}\right\|^{2}+2 \alpha_{n}^{*}\left\|x_{n}-x^{\star}\right\|\left\|x_{n}-x_{n-1}\right\| .
\end{aligned}
$$

Utilizing Lemma 2.1 and Lemma 2.4, we arrive at

$$
\begin{aligned}
\left\|x_{n+1}-x^{\star}\right\|^{2}= & \left\|\left(I-\beta_{n} \gamma F\right) z_{n}-\left(I-\beta_{n} \gamma F\right) x^{\star}-\beta_{n} \gamma F x^{\star}\right\|^{2} \\
\leq & \left\|\left(I-\beta_{n} \gamma F\right) z_{n}-\left(I-\beta_{n} \gamma F\right) x^{\star}\right\|^{2}-2 \beta_{n} \gamma\left\langle F x^{\star}, x_{n+1}-x^{\star}\right\rangle \\
\leq & \left(1-\beta_{n} \eta\right)\left\|w_{n}-x^{\star}\right\|^{2}+2 \beta_{n} \gamma\left\langle F x^{\star}, x^{\star}-x_{n+1}\right\rangle \\
\leq & \left(1-\beta_{n} \eta\right)\left\|x_{n}-x^{\star}\right\|^{2}+2 \beta_{n} \gamma\left\langle F x^{\star}, x^{\star}-x_{n+1}\right\rangle \\
& +\left(\alpha_{n}^{*}\right)^{2}\left\|x_{n}-x_{n-1}\right\|^{2}+2 \alpha_{n}^{*}\left\|x_{n}-x^{\star}\right\|\left\|x_{n}-x_{n-1}\right\| \\
\leq & \left(1-\beta_{n} \eta\right)\left\|x_{n}-x^{\star}\right\|^{2}+\beta_{n} \eta \frac{2 \gamma}{\eta}\left\langle F x^{\star}, x^{\star}-x_{n+1}\right\rangle \\
& +\alpha_{n}^{*}\left\|x_{n}-x_{n-1}\right\|\left(\alpha_{n}^{*}\left\|x_{n}-x_{n-1}\right\|+2\left\|x_{n}-x^{\star}\right\|\right) \\
\leq & \left(1-\beta_{n} \eta\right)\left\|x_{n}-x^{\star}\right\|^{2}+\beta_{n} \eta \frac{2 \gamma}{\eta}\left\langle F x^{\star}, x^{\star}-x_{n+1}\right\rangle+3 \alpha_{n}^{*}\left\|x_{n}-x_{n-1}\right\| M_{2} \\
= & \left(1-\sigma_{n}\right)\left\|x_{n}-x^{\star}\right\|^{2}+\sigma_{n} \vartheta_{n},
\end{aligned}
$$

where

$$
\sigma_{n}=\beta_{n} \eta, \quad \vartheta_{n}=\frac{2 \gamma}{\eta}\left\langle F x^{\star}, x^{\star}-x_{n+1}\right\rangle+\frac{3 \alpha_{n}^{*}}{\beta_{n}} \frac{M_{2}}{\eta}\left\|x_{n}-x_{n-1}\right\|,
$$

and $M_{2}=\sup _{n \in \mathbb{N}}\left\{\left\|x_{n}-x^{\star}\right\|, \alpha_{n}^{*}\left\|x_{n}-x_{n-1}\right\|\right\}$. It is easy to see that $\sigma_{n} \rightarrow 0$ and $\sum_{n=1}^{\infty} \sigma_{n}=\infty$. Since $\left\{x_{n}\right\}$ is bounded, there exists a constant $M_{3}>0$ such that $2 \gamma\left\langle F x^{\star}, x^{\star}-x_{n+1}\right\rangle \leq M_{3}$. From algorithm (3.1), Lemma (2.1) and Lemma (2.4), we have

$$
\begin{aligned}
\left\|x_{n+1}-x^{\star}\right\|^{2} & =\left\|\left(I-\beta_{n} \gamma F\right) z_{n}-\left(I-\beta_{n} \gamma F\right) x^{\star}-\beta_{n} \gamma F x^{\star}\right\|^{2} \\
& \leq\left\|\left(I-\beta_{n} \gamma F\right) z_{n}-\left(I-\beta_{n} \gamma F\right) x^{\star}\right\|^{2}-2 \beta_{n} \gamma\left\langle F x^{\star}, x_{n+1}-x^{\star}\right\rangle \\
& \leq\left(1-\beta_{n} \eta\right)^{2}\left\|z_{n}-x^{\star}\right\|^{2}+2 \beta_{n} \gamma\left\langle F x^{\star}, x^{\star}-x_{n+1}\right\rangle \\
& \leq\left\|z_{n}-x^{\star}\right\|^{2}+\beta_{n} M_{3} .
\end{aligned}
$$

From inequality (3.8) and inequality (3.4), we arrive at

$$
\begin{aligned}
\left\|x_{n+1}-x^{\star}\right\|^{2} \leq & \left\|w_{n}-x^{\star}\right\|^{2}-\sum_{i=1}^{m} a^{(i)} \theta_{n}^{(i)}\left(\frac{2 l^{(i)}}{\zeta^{(i)}}-\theta_{n}^{(i)}\right)\left\|T^{(i)} w_{n}-w_{n}\right\|^{2} \\
& -\sum_{j=1}^{k} b^{(j)} \delta_{n}^{(j)}\left(\frac{2 r^{(j)}}{\vartheta^{(j)}}-\delta_{n}^{(j)}\right)\left\|S^{(j)} y_{n}-y_{n}\right\|^{2}+\beta_{n} M_{3} .
\end{aligned}
$$


By using inequality (3.6), we obtain

$$
\begin{aligned}
\left\|w_{n}-x^{\star}\right\|^{2} & \leq\left(\left\|x_{n}-x^{\star}\right\|+\beta_{n} M_{1}\right)^{2} \\
& =\left\|x_{n}-x^{\star}\right\|+\beta_{n}\left(2 M_{1}\left\|x_{n}-x^{\star}\right\|+\beta_{n} M_{1}^{2}\right) \\
& \leq\left\|x_{n}-x^{\star}\right\|^{2}+\beta_{n} M_{4}
\end{aligned}
$$

for some $M_{4}>0$. From inequality (3.9) and the inequality (3.10), we obtain that

$$
\begin{aligned}
\left\|x_{n+1}-x^{\star}\right\|^{2} \leq & \left\|x_{n}-x^{\star}\right\|^{2}+\beta_{n} M_{3}-\sum_{i=1}^{m} a^{(i)} \theta_{n}^{(i)}\left(\frac{2 l^{(i)}}{\zeta^{(i)}}-\theta_{n}^{(i)}\right)\left\|T^{(i)} w_{n}-w_{n}\right\|^{2} \\
& -\sum_{j=1}^{k} b^{(j)} \delta_{n}^{(j)}\left(\frac{2 r^{(j)}}{\vartheta(j)}-\delta_{n}^{(j)}\right)\left\|S^{(j)} y_{n}-y_{n}\right\|^{2}+\beta_{n} M_{4} .
\end{aligned}
$$

Setting

$$
\begin{aligned}
\xi_{n}= & \sum_{i=1}^{m} a^{(i)} \theta_{n}^{(i)}\left(\frac{2 l^{(i)}}{\zeta^{(i)}}-\theta_{n}^{(i)}\right)\left\|T^{(i)} w_{n}-w_{n}\right\|^{2} \\
& +\sum_{j=1}^{k} b^{(j)} \delta_{n}^{(j)}\left(\frac{2 r^{(j)}}{\vartheta^{(j)}}-\delta_{n}^{(j)}\right)\left\|S^{(j)} y_{n}-y_{n}\right\|^{2},
\end{aligned}
$$

and

$$
\zeta_{n}=\beta_{n}\left(M_{3}+M_{4}\right), \quad s_{n}=\left\|x_{n}-x^{\star}\right\|^{2},
$$

we have that (3.11) can be rewritten in the following form:

$$
s_{n+1} \leq s_{n}-\xi_{n}+\zeta_{n}
$$

To use Lemma 2.5, (consider inequalities (3.7) and (3.12)), it suffices to verify that, for all subsequences $\left\{n_{k}\right\} \subset\{n\}, \lim _{k \rightarrow \infty} \xi_{n_{k}}=0$ implies $\lim \sup _{k \rightarrow \infty} \delta_{n_{k}} \leq 0$. We assume that $\lim _{k \rightarrow \infty} \xi_{n_{k}}=$ 0 . From our assumption we obtain

$$
\lim _{k \rightarrow \infty}\left\|T^{(i)} w_{n_{k}}-w_{n_{k}}\right\|=\lim _{k \rightarrow \infty}\left\|S^{(j)} y_{n_{k}}-y_{n_{k}}\right\|=0 .
$$

Note that

$$
\left\|x_{n}-w_{n}\right\|=\alpha_{n}^{*}\left\|x_{n}-x_{n-1}\right\|=\beta_{n} \frac{\alpha_{n}^{*}}{\beta_{n}}\left\|x_{n}-x_{n-1}\right\| \rightarrow 0 .
$$

From (3.13), we have

$$
\left\|y_{n_{k}}-w_{n_{k}}\right\|=\left\|\sum_{i=1}^{m} a^{(i)} l^{(i)} \theta_{n}^{(i)}\left(T^{(i)}-I\right) w_{n_{k}}\right\| \rightarrow 0 .
$$

This implies that $\left\|y_{n_{k}}-x_{n_{k}}\right\| \leq\left\|y_{n_{k}}-w_{n_{k}}\right\|+\left\|w_{n_{k}}-x_{n_{k}}\right\| \rightarrow 0$. In view of (3.13), we obtain

$$
\left\|z_{n_{k}}-y_{n_{k}}\right\|=\left\|\sum_{j=1}^{k} b^{(j)} r^{(j)} \delta_{n}^{(j)}\left(S^{(j)}-I\right) y_{n_{k}}\right\| \rightarrow 0 .
$$

Hence $\left\|z_{n_{k}}-x_{n_{k}}\right\| \leq\left\|z_{n_{k}}-y_{n_{k}}\right\|+\left\|y_{n_{k}}-x_{n_{k}}\right\| \rightarrow 0$. Since $\left\{x_{n_{k}}\right\}$ is bounded, there exists a subsequence $\left\{x_{n_{k_{j}}}\right\}$ of $\left\{x_{n_{k}}\right\}$, which converges weakly to $\widehat{x}$. Without loss of generality, we can assume that $x_{n_{k}} \rightarrow \widehat{x}$. Since $\lim _{k \rightarrow \infty}\left\|y_{n_{k}}-x_{n_{k}}\right\|=0$, we have $y_{n_{k}} \rightarrow \widehat{x}$. Now from (3.13) and the demiclosedness of $I-T^{(i)}$, we have $\widehat{x} \in \bigcap_{i=1}^{m} F i x\left(T^{(i)}\right)$. By similar argument, we obtain $\widehat{x} \in \bigcap_{j=1}^{k} \operatorname{Fix}\left(S^{(j)}\right)$. Thus $\widehat{x} \in \Omega$. 
Next, we show that $\limsup _{k \rightarrow \infty}\left\langle F x^{\star}, x^{\star}-x_{n_{k}}\right\rangle \leq 0$. To show this inequality, we choose a subsequence $\left\{x_{n_{k_{j}}}\right\}$ of $\left\{x_{n_{k}}\right\}$ such that

$$
\lim _{j \rightarrow \infty}\left\langle F x^{\star}, x^{\star}-x_{n_{k_{j}}}\right\rangle=\limsup _{k \rightarrow \infty}\left\langle F x^{\star}, x^{\star}-x_{n_{k}}\right\rangle .
$$

Since $x^{\star}$ is the unique solution of $\operatorname{VI}(\Omega, F), \widehat{x} \in \Omega$, and $\left\{x_{n_{k_{j}}}\right\}$ converges weakly to $\widehat{x} \in \Omega$, we conclude that

$$
\limsup _{k \rightarrow \infty}\left\langle F x^{\star}, x^{\star}-x_{n_{k}}\right\rangle=\lim _{j \rightarrow \infty}\left\langle F x^{\star}, x^{\star}-x_{n_{k_{j}}}\right\rangle=\left\langle F x^{\star}, x^{\star}-\widehat{x}\right\rangle \leq 0 .
$$

Therefore $\limsup _{k \rightarrow \infty} \vartheta_{n_{k}} \leq 0$. Hence, all conditions of Lemma 2.5 are satisfied. Therefore, we immediately deduce that $\lim _{n \rightarrow \infty} s_{n}=\lim _{n \rightarrow \infty}\left\|x_{n}-x^{\star}\right\|^{2}=0$, that is, $\left\{x_{n}\right\}$ converges strongly to $x^{\star}$, which is the unique solution of $\operatorname{VI}(\Omega, F)$.

Theorem 3.2. Let $\mathscr{H}$ be a Hilbert space. Let, for each $i \in\{1,2, \ldots, m\}, t^{(i)} \in[0,1)$ and $T^{(i)}$ : $\mathscr{H} \rightarrow \mathscr{H}$ be a $t^{(i)}$-strictly pseudo-contractive mapping. Let, for each $j \in\{1,2, \ldots, k\}, L^{(j)} \in$ $(0, \infty)$ and $U^{(j)}: \mathscr{H} \rightarrow \mathscr{H}$ be a $L^{(j)}$-Lipschitzian mapping. Suppose that $\Omega=\bigcap_{i=1}^{m} F i x\left(T^{(i)}\right) \cap$ $\bigcap_{j=1}^{k} F i x\left(\frac{U^{(j)}}{L^{(j)}}\right) \neq \emptyset$. Let the operator $F: \mathscr{H} \rightarrow \mathscr{H}$ be l-Lipschitz continuous and $\delta$-strongly monotone with constants $l>0$ and $\delta>0$. Assume that $\gamma \in\left(0, \frac{2 \delta}{l^{2}}\right)$ and $\alpha^{*}>0$. Let $\left\{x_{n}\right\}$ be a sequence defined by

$$
\left\{\begin{array}{l}
x_{1}, x_{0} \in \mathscr{H} \text { is chosen arbitrarily, } \\
w_{n}=x_{n}+\alpha_{n}^{*}\left(x_{n}-x_{n-1}\right), \\
y_{n}=w_{n}+\sum_{i=1}^{m} a^{(i)} \theta_{n}^{(i)}\left(T^{(i)} w_{n}-w_{n}\right) \\
z_{n}=y_{n}-\sum_{j=1}^{k} b^{(j)} \delta_{n}^{(j)}\left(\left(L^{(j)} I-U^{(j)}\right) y_{n}\right) \\
x_{n+1}=\left(I-\gamma \beta_{n} F\right) z_{n}, \quad \forall n \geq 1,
\end{array}\right.
$$

where $0 \leq \alpha_{n}^{*} \leq \bar{\alpha}_{n}$ such that

$$
\bar{\alpha}_{n}=\left\{\begin{array}{l}
\min \left\{\frac{\varepsilon_{n}}{\left\|x_{n}-x_{n-1}\right\|}, \alpha^{*}\right\}, \quad x_{n} \neq x_{n-1} \\
\alpha^{*}, \quad \text { otherwise. }
\end{array}\right.
$$

Let the sequences $\left\{\beta_{n}\right\},\left\{a^{(i)}\right\},\left\{b^{(j)}\right\},\left\{\theta_{n}^{(i)}\right\}$, and $\left\{\delta_{n}^{(j)}\right\}$ satisfy the following conditions

(i) $\left\{\beta_{n}\right\} \subset(0,1), \lim _{n \rightarrow \infty} \beta_{n}=0$ and $\sum_{n=0}^{\infty} \beta_{n}=\infty$;

(ii) $a^{(i)}, b^{(j)} \in(0,1]$ and $\sum_{i=1}^{m} a^{(i)}=\sum_{j=1}^{k} b^{(j)}=1$;

(iii) $0<c^{(i)} \leq \theta_{n}^{(i)}<1-t^{(i)}$, for $i \in\{1,2, \ldots, m\}$;

(iv) $0<d^{(j)} \leq \delta_{n}^{(j)}<\frac{2}{L^{(j)}}$ for $j \in\{1,2, \ldots, k\}$;

(v) $\varepsilon_{n}>0$ and $\lim _{n \rightarrow \infty} \frac{\varepsilon_{n}}{\beta_{n}}=0$.

Then, $\left\{x_{n}\right\}$ converges strongly to the unique solution $x^{\star} \in V I(\Omega, F)$.

Proof. Since $T^{(i)}$ is a $t^{(i)}$-strict pseudo-contraction with $\operatorname{Fix}\left(T^{(i)}\right) \neq \emptyset$, we have $T^{(i)}$ is $\frac{2}{1-t^{(i)}}$ generalized demimetric. Since $U^{(j)}: \mathscr{H} \rightarrow \mathscr{H}$ is $L^{(j)}$-Lipschitzian and $F i x\left(\frac{U^{(j)}}{L^{(j)}}\right) \neq \emptyset$, we have that $S^{(j)}=\left(L^{(j)}+1\right) I-U^{(j)}$ is $\left(-L^{(j)}\right)$-generalized demimetric. Furthermore, we know that $I-T^{(j)}$ and $I-S^{(j)}$ are demiclosed at 0 (see [13]). Now utilizing Theorem 3.1, we obtain the desired result. 


\section{Applications}

4.1. The triple hierarchical variational inequality problem. A variational inequality problem defined over the set of solutions of hierarchical variational inequality problem is called a triple hierarchical variational inequality problem (THVIP). Iiduka ([23, 24]) proposed some iterative methods for computing the approximate solutions of THVIP. The strong convergence of the sequences generated by the proposed methods was also studied. Now, as an application of our main results, we propose a new iterative algorithm for solving a class of triple hierarchical variational inequality problems with a finite family of generalized demimetric mappings.

Theorem 4.1. Let $\mathscr{H}$ be a Hilbert space. Let, for each $i \in\{1,2, \ldots, m\}, \zeta^{(i)} \neq 0$ and $T^{(i)}$ : $\mathscr{H} \rightarrow \mathscr{H}$ be a $\zeta^{(i)}$-generalized demimetric mapping such that $I-T^{(i)}$ is demiclosed at 0 . Let, for each $j \in\{1,2, \ldots, k\}, A^{(j)}: \mathscr{H} \rightarrow \mathscr{H}$ be a $\kappa^{(j)}$-inverse-strongly monotone mapping. Suppose that $\Omega=\bigcap_{i=1}^{m} F i x\left(T^{(i)}\right) \bigcap \bigcap_{j=1}^{k}\left(A^{(j)}\right)^{-1}(0) \neq \emptyset$. Let the operator $F: \mathscr{H} \rightarrow \mathscr{H}$ be l-Lipschitz continuous and $\delta$-strongly monotone with constants $l>0$ and $\delta>0$. Assume that $\gamma \in\left(0, \frac{2 \delta}{l^{2}}\right)$ and $\alpha^{*}>0$. Let $\left\{x_{n}\right\}$ be a sequence defined by

$$
\left\{\begin{array}{l}
x_{1}, x_{0} \in \mathscr{H} \text { is chosen arbitrarily, } \\
w_{n}=x_{n}+\alpha_{n}^{*}\left(x_{n}-x_{n-1}\right), \\
y_{n}=w_{n}+\sum_{i=1}^{m} a^{(i)} l^{(i)} \theta_{n}^{(i)}\left(T^{(i)} w_{n}-w_{n}\right) \\
z_{n}=y_{n}-\sum_{j=1}^{k} b^{(j)} \delta_{n}^{(j)}\left(A^{(j)}\right) y_{n}, \\
x_{n+1}=\left(I-\gamma \beta_{n} F\right) z_{n}, \quad \forall n \geq 1,
\end{array}\right.
$$

where $l^{(i)}=\frac{\zeta^{(i)}}{\left|\zeta^{(i)}\right|}$ and $0 \leq \alpha_{n}^{*} \leq \bar{\alpha}_{n}$ such that

$$
\bar{\alpha}_{n}= \begin{cases}\min \left\{\frac{\varepsilon_{n}}{\left\|x_{n}-x_{n-1}\right\|}, \alpha^{*}\right\}, & x_{n} \neq x_{n-1} \\ \alpha^{*}, & \text { otherwise. }\end{cases}
$$

Let the sequences $\left\{\beta_{n}\right\},\left\{a^{(i)}\right\},\left\{b^{(j)}\right\},\left\{\theta_{n}^{(i)}\right\}$, and $\left\{\delta_{n}^{(j)}\right\}$ satisfy the following conditions

(i) $\left\{\beta_{n}\right\} \subset(0,1), \lim _{n \rightarrow \infty} \beta_{n}=0$ and $\sum_{n=0}^{\infty} \beta_{n}=\infty$;

(ii) $a^{(i)}, b^{(j)} \in(0,1]$ and $\sum_{i=1}^{m} a^{(i)}=\sum_{j=1}^{k} b^{(j)}=1$;

(iii) $0<c^{(i)} \leq \theta_{n}^{(i)}<2 \frac{l^{(i)}}{\zeta^{(i)}}$, for $i \in\{1,2, \ldots, m\}$;

(iv) $0<d^{(j)} \leq \delta_{n}^{(j)}<2 \kappa^{(j)}$ for $j \in\{1,2, \ldots, k\}$;

(v) $\varepsilon_{n}>0$ and $\lim _{n \rightarrow \infty} \frac{\varepsilon_{n}}{\beta_{n}}=0$.

Then, the $\left\{x_{n}\right\}$ converges strongly to the unique solution $x^{\star} \in \operatorname{VI}(\Omega, F)$.

Proof. Since, for each $j \in\{1,2, \ldots, k\}, A^{(j)}: \mathscr{H} \rightarrow \mathscr{H}$ is a $\kappa^{(j)}$-inverse-strongly monotone mapping, we know from [13] that $S^{(j)}=A^{(j)}+I$ is $\left(\frac{-1}{\kappa^{(j)}}\right)$-generalized demimetric and $I-S^{(j)}$ is demiclosed at 0 . In view of $\left(A^{(j)}\right)^{-1}(0)=F i x\left(S^{(j)}\right)$, we obtain the desired result immediately by following the proof of Theorem 3.1.

Corollary 4.1. Let $\mathscr{H}$ be a Hilbert space. Let, for each $i \in\{1,2, \ldots, m\}, T^{(i)}: \mathscr{H} \rightarrow \mathscr{H}$ be a nonexpansive mapping. Let $A: \mathscr{H} \rightarrow \mathscr{H}$ be a $\kappa$-inverse-strongly monotone mapping. Suppose that $\Omega=\bigcap_{i=1}^{m} \operatorname{Fix}\left(T^{(i)}\right) \bigcap A^{-1}(0) \neq \emptyset$. Let the operator $F: \mathscr{H} \rightarrow \mathscr{H}$ be l-Lipschitz continuous 
and $\delta$-strongly monotone with constants $l>0$ and $\delta>0$. Assume that $\gamma \in\left(0, \frac{2 \delta}{l^{2}}\right)$ and $\alpha^{*}>0$. Let $\left\{x_{n}\right\}$ be a sequence defined by

$$
\left\{\begin{array}{l}
x_{1}, x_{0} \in \mathscr{H} \text { is chosen arbitrarily, } \\
w_{n}=x_{n}+\alpha_{n}^{*}\left(x_{n}-x_{n-1}\right), \\
y_{n}=w_{n}+\sum_{i=1}^{m} a^{(i)} l^{(i)} \theta_{n}^{(i)}\left(T^{(i)} w_{n}-w_{n}\right) \\
z_{n}=\left(I-\delta_{n} A\right) y_{n}, \\
x_{n+1}=\left(I-\gamma \beta_{n} F\right) z_{n}, \quad n \geq 1,
\end{array}\right.
$$

where $0 \leq \alpha_{n}^{*} \leq \bar{\alpha}_{n}$ such that

$$
\bar{\alpha}_{n}= \begin{cases}\min \left\{\frac{\varepsilon_{n}}{\left\|x_{n}-x_{n-1}\right\|},\right. & \left.\alpha^{*}\right\}, \quad x_{n} \neq x_{n-1} \\ \alpha^{*}, & \text { otherwise. }\end{cases}
$$

Let the sequences $\left\{\beta_{n}\right\},\left\{a^{(i)}\right\},\left\{\theta_{n}^{(i)}\right\}$ and $\left\{\delta_{n}\right\}$ satisfy the following conditions:

(i) $\left\{\beta_{n}\right\} \subset(0,1), \lim _{n \rightarrow \infty} \beta_{n}=0$ and $\sum_{n=0}^{\infty} \beta_{n}=\infty$;

(ii) $a^{(i)} \in(0,1]$ and $\sum_{i=1}^{m} a^{(i)}=1$;

(iii) $0<c^{(i)} \leq \theta_{n}^{(i)}<1$, for $i \in\{1,2, \ldots, m\}$;

(iv) $0<d \leq \delta_{n}<2 \kappa$;

(v) $\varepsilon_{n}>0$ and $\lim _{n \rightarrow \infty} \frac{\varepsilon_{n}}{\beta_{n}}=0$.

Then, the sequence $\left\{x_{n}\right\}$ converges strongly to the unique solution $x^{\star} \in \operatorname{VI}\left(\operatorname{VI}\left(\bigcap_{i=1}^{m} F i x\left(T^{(i)}\right), A\right)\right.$, $F)$.

Proof. Since $T^{(i)}$ is a nonexpansive mapping, we have that $T^{(i)}$ is 2-generalized demimetric. Furthermore,we know that $I-T^{(i)}$ is demiclosed at 0. Now utilizing Lemma 2.6 and Theorem 4.1, we obtain the desired result immediately.

4.2. The triple-hierarchical constrained optimization problem. A function, $f: \mathscr{H} \rightarrow \mathbb{R}$ is said to be convex if, for any $x, y \in \mathscr{H}$ and for any $\lambda \in[0,1], f(\lambda x+(1-\lambda) y) \leq \lambda f(x)+(1-$ $\lambda) f(y)$. In particular, a convex function $f: \mathscr{H} \rightarrow \mathbb{R}$ is said to be strongly convex with $c>0$ (c-strongly convex) if

$$
f(\lambda x+(1-\lambda) y) \leq \lambda f(x)+(1-\lambda) f(y)-\frac{c \lambda(1-\lambda)}{2}\|x-y\|^{2},
$$

for all $x, y \in \mathscr{H}$ and for all $\lambda \in[0,1]$.

Let $f: \mathscr{H} \rightarrow \mathbb{R}$ be a Fréchet differentiable function. If $f$ is convex (resp. $c$-strongly convex), then $\nabla f$ is monotone (resp. $c$-strongly monotone). If $f$ is convex and $\nabla f$ is $L$-Lipschitz continuous, then $\nabla f$ is $\frac{1}{L}$-inverse-strongly monotone.

Now we present a new algorithm for the triple-hierarchical constrained optimization problem.

Theorem 4.2. Let $\mathscr{H}$ be a Hilbert space. Let, for each $i \in\{1,2, \ldots, m\}, \zeta^{(i)} \neq 0$ and $T^{(i)}$ : $\mathscr{H} \rightarrow \mathscr{H}$ be a $\zeta^{(i)}$-generalized demimetric mapping such that $I-T^{(i)}$ is demiclosed at 0 . Let, for each $j \in\{1,2, \ldots, k\}, \psi^{(j)}: \mathscr{H} \rightarrow \mathbb{R}$ be a convex and Fréchet differentiable function and $\nabla \psi^{(j)}$ is $L^{(j)}$-Lipschitz continuous. Suppose that $\Omega=\bigcap_{i=1}^{m} \operatorname{Fix}\left(T^{(i)}\right) \cap \bigcap_{j=1}^{k}\left(\nabla \psi^{(j)}\right)^{-1}(0) \neq \emptyset$. 
Let the operator $\Phi: \mathscr{H} \rightarrow \mathbb{R}$ be a $\delta$-strongly convex and Fréchet differentiable, and $\nabla \Phi$ is l-Lipschitz continuous. Assume that $\gamma \in\left(0, \frac{2 \delta}{l^{2}}\right)$ and $\alpha^{*}>0$. Let $\left\{x_{n}\right\}$ be a sequence defined by

$$
\left\{\begin{array}{l}
x_{1}, x_{0} \in \mathscr{H} \text { is chosen arbitrarily, } \\
w_{n}=x_{n}+\alpha_{n}^{*}\left(x_{n}-x_{n-1}\right), \\
y_{n}=w_{n}+\sum_{i=1}^{m} a^{(i)} l^{(i)} \theta_{n}^{(i)}\left(T^{(i)} w_{n}-w_{n}\right) \\
z_{n}=y_{n}-\sum_{j=1}^{k} b^{(j)} \delta_{n}^{(j)}\left(\nabla \psi^{(j)}\right) y_{n}, \\
x_{n+1}=\left(I-\gamma \beta_{n} \nabla \Phi\right) z_{n}, \quad n \geq 0,
\end{array}\right.
$$

where $l^{(i)}=\frac{\zeta^{(i)}}{\left|\zeta^{(i)}\right|}$ and $0 \leq \alpha_{n}^{*} \leq \bar{\alpha}_{n}$ such that

$$
\bar{\alpha}_{n}= \begin{cases}\min \left\{\frac{\varepsilon_{n}}{\left\|x_{n}-x_{n-1}\right\|}, \alpha^{*}\right\}, & x_{n} \neq x_{n-1} \\ \alpha^{*}, & \text { otherwise. }\end{cases}
$$

Let the sequences $\left\{\beta_{n}\right\},\left\{a^{(i)}\right\},\left\{b^{(j)}\right\},\left\{\theta_{n}^{(i)}\right\}$, and $\left\{\delta_{n}^{(j)}\right\}$ satisfy the following conditions

(i) $\left\{\beta_{n}\right\} \subset(0,1), \lim _{n \rightarrow \infty} \beta_{n}=0$ and $\sum_{n=0}^{\infty} \beta_{n}=\infty$;

(ii) $a^{(i)}, b^{(j)} \in(0,1]$ and $\sum_{i=1}^{m} a^{(i)}=\sum_{j=1}^{k} b^{(j)}=1$;

(iii) $0<c^{(i)} \leq \theta_{n}^{(i)}<2 \frac{l^{(i)}}{\zeta^{(i)}}$, for $i \in\{1,2, \ldots, m\}$;

(iv) $0<d^{(j)} \leq \delta_{n}^{(j)}<\frac{2}{L^{(j)}}$ for $j \in\{1,2, \ldots, k\}$;

(v) $\varepsilon_{n}>0$ and $\lim _{n \rightarrow \infty} \frac{\varepsilon_{n}}{\beta_{n}}=0$.

Then, $\left\{x_{n}\right\}$ converges strongly to the unique solution $x^{\star} \in V I(\Omega, \nabla F)$.

Proof. We note that $\nabla \psi^{(j)}$ is $\frac{1}{L^{(j)}}$-inverse-strongly monotone and $\nabla \Phi$ is $l$-Lipschitz $\delta$-strongly monotone mapping. Putting $A^{(j)}=\nabla \psi^{(j)}$ and $F=\nabla \Phi$ in Theorem 4.1, we have from Theorem 4.1 the desired result immediately.

4.3. The hierarchical minimization problem. Suppose that $\Phi: \mathscr{H} \rightarrow \mathbb{R}$ is $\delta$-strongly convex and Fréchet differentiable, and $\nabla \Phi: \mathscr{H} \rightarrow \mathscr{H}$ is $l$-Lipschitz continuous. Let $C$ be a nonempty closed convex subset of $\mathscr{H}$. Then, $\operatorname{VI}(C, \nabla \Phi)$ can be characterized as the set of all minimizers of $\Phi$ over $C$

$$
V I(C, \nabla \Phi)=\arg \min _{x \in C} \Phi(x):=\left\{x^{*} \in C: \Phi\left(x^{*}\right)=\min _{x \in C} \Phi(x)\right\}
$$

Now setting $S^{(i)}=I$ in Theorem 3.1, we obtain the following result.

Theorem 4.3. Let $\mathscr{H}$ be a Hilbert space. Let, for each $i \in\{1,2, \ldots, m\}, \zeta^{(i)} \neq 0$ and $T^{(i)}: \mathscr{H} \rightarrow$ $\mathscr{H}$ be a $\zeta^{(i)}$-generalized demimetric mapping such that $I-T^{(i)}$ is demiclosed at 0 . Suppose that $\Omega=\bigcap_{i=1}^{m} \operatorname{Fix}\left(T^{(i)}\right) \neq \emptyset$. Let the operator $\Phi: \mathscr{H} \rightarrow \mathbb{R}$ be a $\delta$-strongly convex and Fréchet differentiable, and $\nabla \Phi$ is l-Lipschitz continuous. Assume that $\gamma \in\left(0, \frac{2 \delta}{l^{2}}\right)$ and $\alpha^{*}>0$. Let $\left\{x_{n}\right\}$ 
be a sequence defined by

$$
\left\{\begin{array}{l}
x_{1}, x_{0} \in \mathscr{H} \text { is chosen arbitrarily, } \\
w_{n}=x_{n}+\alpha_{n}^{*}\left(x_{n}-x_{n-1}\right) \\
y_{n}=w_{n}+\sum_{i=1}^{m} a^{(i)} l^{(i)} \theta_{n}^{(i)}\left(T^{(i)} w_{n}-w_{n}\right) \\
x_{n+1}=\left(I-\gamma \beta_{n} \nabla \Phi\right) y_{n}, \quad n \geq 1,
\end{array}\right.
$$

where $l^{(i)}=\frac{\zeta^{(i)}}{\left|\zeta^{(i)}\right|}$ and $0 \leq \alpha_{n}^{*} \leq \bar{\alpha}_{n}$ such that

$$
\bar{\alpha}_{n}=\left\{\begin{array}{l}
\min \left\{\frac{\varepsilon_{n}}{\left\|x_{n}-x_{n-1}\right\|}, \alpha^{*}\right\}, \quad x_{n} \neq x_{n-1} \\
\alpha^{*}, \quad \text { otherwise. }
\end{array}\right.
$$

Let the sequences $\left\{\beta_{n}\right\},\left\{a^{(i)}\right\}$, and $\left\{\theta_{n}^{(i)}\right\}$ satisfy the following conditions

(i) $\left\{\beta_{n}\right\} \subset(0,1), \lim _{n \rightarrow \infty} \beta_{n}=0$ and $\sum_{n=0}^{\infty} \beta_{n}=\infty$;

(ii) $a^{(i)} \in(0,1]$ and $\sum_{i=1}^{m} a^{(i)}=1$;

(iii) $0<c^{(i)} \leq \theta_{n}^{(i)}<2 \frac{l^{(i)}}{\zeta^{(i)}}$, for $i \in\{1,2, \ldots, m\}$;

(iv) $\varepsilon_{n}>0$ and $\lim _{n \rightarrow \infty} \frac{\varepsilon_{n}}{\beta_{n}}=0$.

Then, $\left\{x_{n}\right\}$ converges strongly to the unique solution $x^{\star} \in \arg \min _{F i x\left(T^{(i)}\right)} \Phi$.

4.4. The generalized multiple-set split feasibility problem. Now we consider the following generalized multiple-set split feasibility problem in Hilbert spaces. Let $\mathscr{H}, \mathscr{H}^{(j)}, j=$ $1,2, \ldots, k$, be real Hilbert spaces and let $B^{(j)}: \mathscr{H} \rightarrow \mathscr{H}^{(j)}, j=1,2, \ldots, k$, be bounded linear operators. Let, for each $i \in\{1,2, \ldots, m\}, C^{(i)}$ be a nonempty closed convex subset of $\mathscr{H}$. Let, for each $j \in\{1,2, \ldots, k\}, Q^{(j)}$ be a nonempty closed convex subset of $\mathscr{H}^{(j)}$. Then the generalized multiple-set split feasibility problem (GMSSFP) is to find an element $x^{\star}$ such that

$$
x^{\star} \in \Omega=\left(\bigcap_{i=1}^{m} C^{(i)}\right) \bigcap\left(\bigcap_{j=1}^{k}\left(B^{(j)}\right)^{-1}\left(Q^{(j)}\right)\right),
$$

that is, $x^{\star} \in \bigcap_{i=1}^{m} C^{(i)}$ and $B^{(j)} x^{\star} \in Q^{(j)}$ for each $j=1,2, \ldots, k$.

The (GMSSFP) with $m=k=1$ is known as the split feasibility problem (introduced by Censor and Elfving (1994) [25]). The (GMSSFP) with $\mathscr{H}^{(1)}=\ldots=\mathscr{H}^{(k)}$ and $B^{(1)}=\ldots=$ $B^{(k)}$ is known as the multiple-set split feasibility problem (introduced by Censor et al. (2005) [26]). The (GMSSFP) with $m=1$ is known as the split feasibility problem with multiple output sets (introduced by Reich et al. [27]). These problems are the core of the modeling of many inverse problems in various areas of mathematics and physical sciences, and has been used to model significant real-world inverse problems in sensor networks, radiation therapy treatment planning, and computerized tomography (see, e.g., [26, 28, 29]).

Theorem 4.4. Let $\mathscr{H}, \mathscr{H}^{(j)}, j=1,2, \ldots, k$, be real Hilbert spaces. Let, for each $j \in\{1,2, \ldots, k\}$, $Q^{(j)}$ be a nonempty closed convex subset of $\mathscr{H}^{(j)}$. Let $B^{(j)}: \mathscr{H} \rightarrow \mathscr{H}^{(j)}$ be a bounded linear operator and $B^{(j)^{*}}$ be the adjoint of $B^{(j)}$. Let, for each $i \in\{1,2, \ldots, m\}, C^{(i)}$ be a nonempty closed convex subset of $\mathscr{H}$. Assume that $\Omega=\left(\bigcap_{i=1}^{m} C^{(i)}\right) \cap\left(\bigcap_{j=1}^{k}\left(B^{(j)}\right)^{-1}\left(Q^{(j)}\right)\right) \neq \emptyset$. Let the 
operator $F: \mathscr{H} \rightarrow \mathscr{H}$ be l-Lipschitz continuous and $\delta$-strongly monotone with constants $l>0$ and $\delta>0$. Assume that $\gamma \in\left(0, \frac{2 \delta}{l^{2}}\right)$ and $\alpha^{*}>0$. Let $\left\{x_{n}\right\}$ be a sequence defined by

$$
\left\{\begin{array}{l}
x_{1}, x_{0} \in \mathscr{H} \text { is chosen arbitrarily, } \\
w_{n}=x_{n}+\alpha_{n}^{*}\left(x_{n}-x_{n-1}\right), \\
y_{n}=\sum_{i=1}^{m} a^{(i)} P_{C^{(i)}} w_{n} \\
z_{n}=y_{n}-\sum_{j=1}^{k} b^{(j)} \delta_{n}^{(j)}\left(B^{(j)^{*}}\left(I-P_{\left.Q^{(j)}\right)} B^{(j)}\right) y_{n},\right. \\
x_{n+1}=\left(I-\gamma \beta_{n} F\right) z_{n}, \quad n \geq 1,
\end{array}\right.
$$

where and $0 \leq \alpha_{n}^{*} \leq \bar{\alpha}_{n}$ such that

$$
\bar{\alpha}_{n}=\left\{\begin{array}{l}
\min \left\{\frac{\varepsilon_{n}}{\left\|x_{n}-x_{n-1}\right\|}, \alpha^{*}\right\}, \quad x_{n} \neq x_{n-1} \\
\alpha^{*}, \quad \text { otherwise. }
\end{array}\right.
$$

Let the sequences $\left\{\beta_{n}\right\},\left\{a^{(i)}\right\},\left\{b^{(j)}\right\}$, and $\left\{\delta_{n}^{(j)}\right\}$ satisfy the following conditions

(i) $\left\{\beta_{n}\right\} \subset(0,1), \lim _{n \rightarrow \infty} \beta_{n}=0$ and $\sum_{n=0}^{\infty} \beta_{n}=\infty$;

(ii) $a^{(i)}, b^{(j)} \in(0,1]$ and $\sum_{i=1}^{m} a^{(i)}=\sum_{j=1}^{k} b^{(j)}=1$;

(iii) $0<d^{(j)} \leq \delta_{n}^{(j)}<\frac{2}{\left\|B^{(j)}\right\|^{2}}$ for $j \in\{1,2, \ldots, k\}$;

(iv) $\varepsilon_{n}>0$ and $\lim _{n \rightarrow \infty} \frac{\varepsilon_{n}}{\beta_{n}}=0$.

Then, $\left\{x_{n}\right\}$ converges strongly to the unique solution $x^{\star} \in V I(\Omega, F)$.

Proof. We note that, for each $i \in\{1,2, \ldots, m\}, F i x\left(P_{C^{(i)}}\right)=C^{(i)}, P_{C^{(i)}}$ is 1 -generalized demimetric and $I-P_{C^{(i)}}$ is demiclosed at 0 (see [13]). Put $A^{(j)}=B^{(j)^{*}}\left(I-P_{Q^{(j)}}\right) B^{(j)}: \mathscr{H} \rightarrow \mathscr{H}$. We know that $A^{(j)}: \mathscr{H} \rightarrow \mathscr{H}$ is $\frac{1}{\left\|B^{(j)}\right\|^{2}}$-inverse-strongly monotone mapping. Putting $\theta_{n}^{(i)}=1$ in Theorem 4.1, we have from Theorem 4.1 the desired result immediately.

Finally, we provide a numerical experiment and show the performance of our proposed iterative algorithm.

Example 4.1. Let $\mathscr{H}=\mathbb{R}$ be the real line. Define $T$ on $\mathbb{R}$ by $T(x)=3 x-1$. Clearly, $x^{\star}=\frac{1}{2}$ is the only fixed point of $T$. We have $T$ is (-2)-generalized demimetric. Indeed, for each $x \in \mathbb{R}$, we have

$$
(-2)\left(x-\frac{1}{2}\right)(1-2 x)=\zeta\left\langle x-x^{\star}, x-T x\right\rangle=\|x-T x\|^{2}=(1-2 x)^{2} .
$$

(Putting $x^{\star}=\frac{1}{2}$ and $x=1$, we see that $T$ is not strictly pseudo-contractive and is not quasinonexpansive.) Let $F=I-x_{0}$ and $S=I$. Then the operator $S$ is nonexpansive and hence is 2-generalized demimetric. Also the $F$ is 1-strongly monotone and 1-Lipschitz continuous. Take $x_{0}=1, \gamma=1, \theta_{n}=\frac{n+1}{2 n+3}, \delta_{n}=1, \beta_{n}=\frac{1}{n+100}$, and $\alpha_{n}^{*}=0$. In the following figure, we can observe that $\left\{x_{n}\right\}$ corresponding to Algorithm 3.1 converges to $\operatorname{Fix}(T) \cap \operatorname{Fix}(S)=\frac{1}{2}$. 


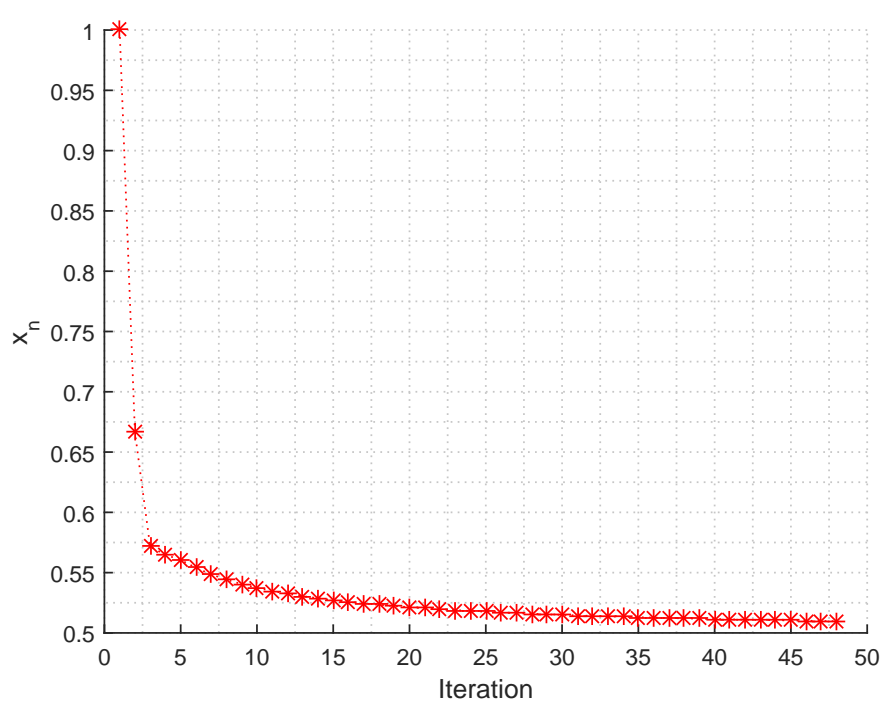

FIGURE 1. The behavior of sequence $\left\{x_{n}\right\}$

\section{REFERENCES}

[1] D. Kinderlehrer, G. Stampacchia, An Introduction to Variational Inequalities and Their Applications, Academic Press, New York, 1980.

[2] F. Facchinei, J.S. Pang, Finite-Dimensional Variational Inequalities and Complementarity Problems, Volume II. Springer Series in Operations Research, Springer, New York, 2003.

[3] M. Tian, M. Tong, A self-adaptive Armijo-like step size method for solving monotone variational inequality problems in Hilbert spaces, J. Nonlinear Funct. Anal. 2019 (2019), Article ID 29.

[4] L.V. Nguyen, X. Qin, The minimal time function associated with a collection of sets, ESAIM Control Optim. Calc. Var. 26 (2020), 93.

[5] T.H. Cuong, J.C. Yao, N.D. Yen, Qualitative properties of the minimum sum-of-squares clustering problem, Optimization, 69 (2020), 2131-2154.

[6] I. Yamada, The hybrid steepest descent method for the variational inequality problems over the intersection of fixed points sets of nonexpansive mappings, In: D. Butnariu, Y. Censor, S. Reich (eds). Inherently Parallel Algorithms in Feasibility and Optimization and Their Application, pp. 473-504, North-Holland, Amsterdam, 2001.

[7] K. Slavakis, I. Yamada, Robust wideband beamforming by the hybrid steepest descent method, IEEE Trans. Signal Process. 55 (2007), 4511-4522.

[8] H. Iiduka, I. Yamada, A use of conjugate gradient direction for the convex optimization problem over the fixed point set of a nonexpansive mapping, SIAM J. Optim. 19 (2009), 1881-1893.

[9] H. Iiduka, Fixed point optimization algorithm and its application to power control in CDMA data networks, Math. Program. 133 (2012), 227-242.

[10] A. Cegielski, Application of quasi-nonexpansive operators to an iterative method for variational inequality, SIAM J Optim. 25 (2015), 2165-2181.

[11] L.C. Ceng, Asymptotic inertial subgradient extragradient approach for pseudomonotone variational inequalities with fixed point constraints of asymptotically nonexpansive mappings, Commun. Optim. Theory 2020 (2020), Article ID 2.

[12] T. Kawasaki, W. Takahashi, A strong convergence theorem for countable families of nonlinear nonself mappings in Hilbert spaces and applications, J. Nonlinear Convex Anal. 19 (2018), 543-560.

[13] W. Takahashi, Weak and strong convergence theorems for new demimetric mappings and the split common fixed point problem in Banach spaces, Numer. Funct. Anal. Optim. 39 (2018), 1011-1033. 
[14] Polyak, B.T: Some methods of speeding up the convergence of iteration methods, USSR Comput. Math. Math. Phys. 4 (1964), 1-17.

[15] D.A. Lorenz, T. Pock, An inertial forward-backward algorithm for monotone inclusions, J. Math. Imaging Vis. 51 (2015), 311-325.

[16] Y. Shehu, A. Gibali, New inertial relaxed method for solving split feasibilities, Optimization, (2020), doi: 10.1007/s11590-020-01603-1.

[17] A. Beck, M. Teboulle, A fast iterative shrinkage-thresholding algorithm for linear inverse problems, SIAM J. Imaging Sci. 2 (2009), 183-202.

[18] B. Tan, S.Y. Cho, Inertial projection and contraction methods for pseudomonotone variational inequalities with non-Lipschitz operators and applications, Appl. Anal. (2021), doi: 10.1080/00036811.2021.1979219 .

[19] J. Fan, L. Liu, X. Qin, A subgradient extragradient algorithm with inertial effects for solving strongly pseudomonotone variational inequalities, Optimization, 69 (2020), 2199-2215.

[20] H.H. Bauschke, P.L. Combettes, Convex Analysis and Monotone Operator Theory in Hilbert Spaces, Springer, Cham, 2011,

[21] S. He, C. Yang, Solving the variational inequality problem defined on intersectoin of finite level sets, Abstr. Appl. Anal. 2013 (2013), Article ID 942315.

[22] L. Liu, X. Qin, R.P. Agarwal, Iterative methods for fixed points and zero points of nonlinear mappings with applications, Optimization, 70 (2021), 693-713.

[23] H. Iiduka, Strong convergence for an iterative method for the triple-hierarchical constrained optimization problem, Nonlinear Anal. 71 (2009), 1292-1297.

[24] H. Iiduka, Iterative algorithm for triple-hierarchical constrained nonconvex optimization problem and its application to network bandwidth allocation, SIAM J. Optim. 22 (2012), 862-878.

[25] Y. Censor, T. Elfving, A multi-projection algorithm using Bregman projections in a product space, Numer. Algo. 8 (1994), 221-239.

[26] Y. Censor, T. Elfving, N. Kopf, T. Bortfeld, The multiple-sets split feasibility problem and its application, Inverse Probl. 21 (2005), 2071-2084.

[27] S. Reich, T.M. Tuyen, T.N.H. Mai, The split feasibility problem withmultiple output sets in Hilbert spaces, Optim. Lett. 14 (2020), 2335-2353.

[28] G. Lopez, V. Martin-Marquez, H.K. Xu, Iterative algorithms for the multiple-sets split feasibility problem, In: Censor, Y., Jiang, M., Wang, G. (eds.) Biomedical Mathematics: Promising Directions in Imaging, Therapy Planning and Inverse Problems, pp. 243-279. Medical Physics Publishing, Madison, 2010.

[29] Y. Censor, S. Petra, C. Schnörr, Superiorization vs. accelerated convex optimization: The superiorized / regularized least-squares case, J. Appl. Numer. Optim. 2 (2020), 15-62. 